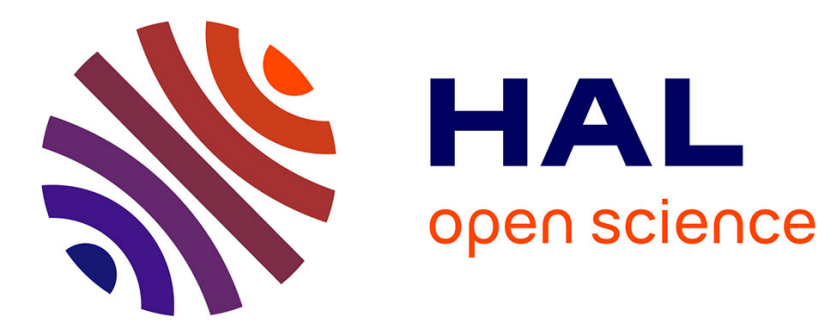

\title{
Dentifrices et vernis fluorés, intérêt dans la prévention des lésions carieuses
}

Michèle Muller Bolla, Sophie Doméjean

\section{To cite this version:}

Michèle Muller Bolla, Sophie Doméjean. Dentifrices et vernis fluorés, intérêt dans la prévention des lésions carieuses. Actualités Pharmaceutiques, 2019, 587, pp.49-53. hal-02326453

\section{HAL Id: hal-02326453 \\ https://hal.science/hal-02326453}

Submitted on 25 Oct 2021

HAL is a multi-disciplinary open access archive for the deposit and dissemination of scientific research documents, whether they are published or not. The documents may come from teaching and research institutions in France or abroad, or from public or private research centers.
L'archive ouverte pluridisciplinaire HAL, est destinée au dépôt et à la diffusion de documents scientifiques de niveau recherche, publiés ou non, émanant des établissements d'enseignement et de recherche français ou étrangers, des laboratoires publics ou privés.

\section{다)(1) $(5$}

Distributed under a Creative Commons Attribution - NonCommerciall 4.0 International 
Dochead pratique

Sous-dochead bucco-dentaire

\title{
Dentifrices et vernis fluorés, intérêt pour la prévention des lésions carieuses
}

\author{
Michèle Muller-Bolla ${ }^{a, *, b, c}$ \\ Professeur des Universités, praticien hospitalier \\ Sophie Doméjean ${ }^{\mathrm{d}, \mathrm{e}, \mathrm{f}}$ \\ Professeur des Universités, praticien hospitalier
}

aUniversité Côte d'Azur, Centre hospitalier universitaire de Nice, Département d'odontologie pédiatrique, 5 rue Pierre-Dévoluy, 06000 Nice, France

'Université de Nice-Sophia Antipolis, UFR d'odontologie, 24 avenue des Diables Bleus, 06357 Nice, France

'URB2i, Faculté de chirurgie dentaire, Université Paris-Descartes, 1 rue Maurice-Arnoux, 92120 Montrouge, France

dDépartement d'odontologie conservatrice, Centre hospitalier universitaire Estaing, Service d'odontologie, 1 place Lucie-Aubrac, 63001 Clermont-Ferrand, France

eUniversité Clermont-Auvergne, UFR d'odontologie, 2 rue de Braga, 63100 Clermont-Ferrand, France ${ }^{f}$ Centre de recherche en odontologie clinique EA 4847, 2 rue de Braga, 63100 Clermont-Ferrand, France

\section{*Auteur correspondant.}

Adresse e-mail : michele.muller@univ-cotedazur.fr (M. Muller-Bolla).

\section{Résumé}

La gestion de la maladie carieuse, chez l'enfant et l'adulte, basée sur l'évaluation du risque carieux et la mise en place d'une prise en charge thérapeutique personnalisée, fait appel à des méthodes de prévention primaire et secondaire. Parmi celles-ci, I'utilisation biquotidienne de dentifrice fluoré à 
domicile et l'application professionnelle de vernis fluoré servent à éviter l'initiation des lésions carieuses ou à favoriser leur reminéralisation.

(C) 2019

Mots clés - dentifrice ; fluor ; maladie carieuse ; prévention ; vernis fluoré

Summary à venir

(c) 2019

Keywords à venir

À l'échelle mondiale, en 2015, la maladie carieuse, qui concernait 2,5 milliards de personnes, constituait la morbidité la plus fréquente. La prévalence des lésions carieuses cavitaires sur dents permanentes non traitées, standardisée sur l'âge, était de $34 \%$. Aussi, contrairement aux idées reçues, la susceptibilité à la maladie carieuse existe tout au long de la vie [1]. En France, la prévalence des dents temporaires non traitées était trois fois plus élevée qu'à l'échelle internationale où elle atteignait déjà 8,7 \% [2]. Il est capital de prendre en charge la maladie carieuse et ses lésions avec des méthodes de prévention primaire et secondaire revisitées selon l'approche contemporaine de cette maladie : l'intervention minimale en cariologie.

\section{T1 Intervention minimale en cariologie}

TEG1 En France, la gestion de la maladie carieuse est basée sur l'évaluation dichotomique du risque carieux : faible ou élevé. Elle repose sur la correction des facteurs pathologiques et le renforcement des facteurs protecteurs (tableau 1) [3], ainsi que sur une prise en charge thérapeutique personnalisée.

TEG1 En complément, des traitements cario-préventifs (prévention primaire) et des traitements non invasifs ou ultraconservateurs (prévention secondaire) des lésions carieuses des dents vitales sont donc indiqués avec le consentement du patient [4]. Les agents fluorés tels que les dentifrices et les vernis font partie des acteurs majeurs en prévention primaire et secondaire.

Tableau 1. Facteurs pris en compte dans la plupart des systèmes d'évaluation du risque carieux individuel $[5,6]$.

Facteurs

Enfants jusqu'à 6 ans

Enfants de plus de

6 ans et adultes

\begin{tabular}{lll}
\hline $\begin{array}{l}\text { Facteurs } \\
\text { prédicteurs }\end{array}$ & Niveau d'éducation des parents \\
& Faible niveau d'éducation
\end{tabular}


Faible niveau socio-économique

Mauvais état de santé bucco-dentaire des

$\mathbf{x}$ parents et de la fratrie ainsi que des personnes s'occupant régulièrement de l'enfant

Expérience carieuse (lésions carieuses, restaurations dentaires récentes)

Petit poids à la naissance

$\mathbf{x}$

Maladies systémiques/handicaps

$x$

\section{Facteurs \\ pathologiques} Biberons nocturnes ou durant la sieste contenant autre chose que de l'eau

Endormissement pendant allaitement prolongé après éruption des incisives

Boissons sucrées (en particulier sodas) en dehors des repas

Bonbons/grignotages à haute teneur en sucres pluriquotidiens

Présence de plaque dentaire/biofilm visible à l'œil nu

Brossage biquotidien absent ou inefficace $\mathbf{x}$ car réalisé par enfant

Brossage biquotidien absent ou inefficace

Brossage au minimum biquotidien (pendant deux minutes à chaque fois), effectué par les parents

Taux salivaire élevé de Streptococcus mutans et de lactobacilles

Flux salivaire diminué (maladie, médicaments...) 
Puits et sillons anfractueux

Exposition radiculaire

Consommation de drogues

Irradiation de la tête et du cou

\begin{tabular}{llll}
\hline $\begin{array}{l}\text { Facteurs } \\
\text { protecteurs }\end{array}$ & $\begin{array}{l}\text { Dentifrice fluoré (dosage adapté à l'âge) } \\
\text { Suivi régulier par un chirurgien-dentiste }\end{array}$ & $\mathbf{x}$ & $\mathbf{x}$ \\
& Consommation quotidienne d'eau fluorée & $\mathbf{x}$ & $\mathbf{x}$
\end{tabular}

Sur 3 colonnes + marge près de son appel

\section{T1 Dentifrice fluoré}

L'utilisation d'un dentifrice fluoré doit être systématisée, quels que soient le risque carieux et l'âge. Ce topique fluoré, d'un coût abordable, joue un rôle majeur dans l'interruption du processus de déminéralisation (lié aux prises alimentaires sucrées quotidiennes et à la consommation de boissons autres que l'eau) et dans l'initiation de la reminéralisation (encadré 1) [3,5,6]. Apporté en petite quantité de manière régulière, il enraye le processus de déminéralisation et potentialise la reminéralisation des tissus dentaires inhérente aux ions calcium et phosphate présents dans la salive : le fluor agit comme catalyseur de fixation de ces ions [7].

\section{Encadré 1. Le processus carieux}

Le processus carieux est le résultat de l'exposition des bactéries cariogènes présentes dans la plaque dentaire et la salive à des glucides fermentescibles qu'elles métabolisent en générant la production d'acides; en deçà du pH 5,5, il y déminéralisation de la couche superficielle de l'émail qui devient poreuse du fait de la perte des minéraux. Dans des conditions physiologiques, dans les 20 minutes qui suivent l'ingestion alimentaire sucrée, la salive, de par son pouvoir tampon et des éléments qui la composent, permet une remontée du pH et la reminéralisation de l'émail par reprécipitation des minéraux [3].

Sur 2 colonnes près de son appel / Insérer l'illustration 1 dans cet encadré

\section{T2 Prévention primaire}


TEG1 L'efficacité des dentifrices fluorés en cario-prévention primaire a été démontrée au début des années 2000 [8]. Une dizaine d'années plus tard, il a été précisé qu'elle dépendait de leur concentration en fluor [9].

TEG1 Exprimée par la fraction préventive (proportion de sujets utilisant quotidiennement un dentifrice fluoré et n'ayant pas développé de lésions carieuses), elle était respectivement de $23 \%$ (19-27; intervalle de confiance [IC] à $95 \%)$ et $36 \%$ (27-44; IC à $95 \%)$ avec les dentifrices fluorés à $1000-1250$ ppm et 2 400-2 800 ppm. En revanche, l'efficacité des dentifrices dosés à moins de 1000 ppm de fluor (ppmF) n'a pas été clairement mise en évidence [10].

\section{T2 Indications, protocole}

TEG1 Les dentifrices à moins de 1000 ppmF ont été abandonnés à partir de l'âge de $\mathbf{2}$ ans dans la plupart des pays au profit de ceux à 1000-1 450 ppmF à la suite de la publication de ces résultats par la Cochrane Library $[8,9]$.

TEG1 En revanche, en France, les recommandations de l'Agence nationale de sécurité du médicament et des produits de santé (Afssaps), basées sur un dosage de moins de 1000 ppmF chez les enfants en denture temporaire, n'ont pas été révisées depuis 2008. Elles avaient été retenues du fait du risque de fluorose dentaire associé à un mauvais contrôle de la déglutition au cours des premières années de la vie [5,11].

TEG1 Cependant, une exception avait déjà été proposée en cas de risque carieux élevé :

le dentifrice à 1000 ppmF pouvait être conseillé, sous réserve de l'absence de consommation d'autres produits fluorés recherchés à l'occasion d'un bilan fluoré (eau, sel, comprimés et gouttes fluorés) et du consentement éclairé des parents [11]. En effet, le risque de fluorose n'est pas à craindre en cas de bonne utilisation du dentifrice à 1000 ppmF chez les enfants de 2 à 6 ans, c'est-àdire, s'il est appliqué par les parents sur la brosse à dents, dans une quantité comparable à la taille d'un petit pois. Certains fabricants ont eu l'idée judicieuse de colorer les touffes de brins centrales des brosses à dents conseillées en denture temporaire pour indiquer la zone de la surface de brossage à recouvrir de dentifrice fluoré (si possible peu goûteux pour qu'il ne soit pas avalé) [12].

TEG1 Les préconisations du Collège des enseignants en odontologie pédiatrique sont les suivantes [12] :

- de l'âge de 6 mois à 2 ans, un brossage biquotidien réalisé par les parents avec un dentifrice à moins de 1000 ppmF (0,1\% d'ion fluor ou $1 \mathrm{mg} / \mathrm{F} / \mathrm{g}$ ) à l'état de trace sur une compresse humide ou un doigt brosse à dents, en remplacement d'une brosse à dents "baby" dont l'utilisation devient la règle avec l'apparition des premières molaires temporaires ;

- de 2 à 6 ans, un brossage biquotidien initialement effectué par un adulte, puis supervisé par celui-ci avec l'acquisition progressive de l'autonomie de l'enfant, réalisé, en cas de risque carieux élevé, avec un dentifrice à 1 000-1 450 ppmF, placé dans le sens de la largeur d'une brosse à dents "enfant", à une quantité équivalente à la taille d'un petit pois (figure 1) ; 
- de 6 à 12 ans, un brossage au minimum biquotidien surveillé et contrôlé par un adulte au moins jusqu'à 8 ans, ou réalisé par l'adulte si l'enfant ou l'adolescent est peu autonome ; la brosse à dents manuelle "junior" ou électrique avec des mouvements dans les trois sens de l'espace (mouvements oscillo-rotatifs ou brosse trizone à pulsations [Oral-B] ou mouvements soniques [Philips]) doit être garnie de dentifrice fluoré sur le tiers à la moitié de la longueur de la surface de brossage rectangulaire [6], ce dernier devant être dosé à 1 000-1 450 ppmF si le risque carieux est faible et à au moins 1500 ppmF (éventuellement 2500 ppmF à partir de l'âge de 10 ans), en cas de risque élevé ;

- à partir de 12 ans, comme chez l'adulte, en complément des consignes précédentes, la brosse à dents "adulte", de taille adaptée, peut être utilisée, mais son action doit être complétée par le passage du fil dentaire dans le but d'améliorer l'élimination de la plaque dentaire au niveau des points de contact interproximaux.

\section{T2 Prévention secondaire}

TEG1 L'utilisation biquotidienne d'un dentifrice fluoré d'au moins 1000 ppmF peut aussi être envisagée comme un traitement curatif non invasif visant la reminéralisation de l'émail et de la dentine. Ce dentifrice peut enrayer le développement des lésions carieuses non cavitaires, sous réserve de la correction des facteurs pathologiques qui sous-tendent le processus de déminéralisation. La lésion carieuse reminéralisée devient souvent noire (figure 2$)^{1}$.

TEG1 Dans les pays ne connaissant aucun problème de démographie professionnelle, le dentifrice d'au moins 1000 ppmF permet également de contrôler les lésions cavitaires des dents temporaires des enfants non coopérants dans l'attente d'un rendez-vous pour traitements ultraconservateurs sous sédation consciente ou anesthésie générale $[13,14]$. En effet, cette solution ne peut être que transitoire du fait de la perte d'espace qu'elle entraînerait et de ses conséquences sur la dentition permanente [12].

\section{T1 Vernis fluoré}

Le vernis est un topique fluoré d'application professionnelle recommandé uniquement chez les patients à risque carieux élevé, quel que soit leur âge (encadré 2).

\section{Encadré 2. À propos des vernis fluorés}

- Haut niveau de preuve de l'efficacité des vernis fluorés en prévention primaire et secondaire.

- Aucune contre-indication liée à l'âge car le risque d'ingestion du fluor est minoré.

- Il n'est pas forcément nécessaire de préparer une cavité avec une fraise, puis de réaliser une restauration pour soigner une lésion carieuse ; les lésions carieuses sont reminéralisables. 


\section{T2 Prévention primaire}

TEG1 L'efficacité cario-préventive des vernis fluorés a été rapportée par une méta-analyse les comparant avec un placebo ou l'absence de traitement chez des sujets de moins de 16 ans. Sa fraction préventive était respectivement de $37 \%$ (24-51; IC $95 \%)$ et $43 \%$ (30-57 ; IC $95 \%$ ) pour les dents temporaires et permanentes. Ce travail, publié en 2013 par la Cochrane Library, a donc conclu que, malgré quelques biais de méthodologie relevés au travers des différentes études (niveau de preuve global modéré), les vernis fluorés représentent une technique de cario-prévention primaire efficace [15]. Aucune incidence significative n'a pas été mise en évidence en fonction du statut carieux initial, de la fréquence d'application (au minimum bi-annuelle) ou de la concentration en fluor du vernis utilisé ( $\geq 22600$ ppm).

TEG1 Plus récemment, une autre méta-analyse a comparé l'efficacité du vernis fluoré à celle du scellement de silllons préventif (autre traitement cario-préventif reconnu dans le cas des molaires permanentes) [16,17]. Elle a souligné une supériorité de l'efficacité cario-préventive des scellements à base de résine composite par rapport au vernis fluoré sur les molaires permanentes, ainsi que l'intérêt d'associer scellement résine et vernis fluoré par rapport aux vernis utilisés seuls.

Cette position a été remise en question en 2017 par des travaux qui concluaient sur une absence de différence significative à 36 mois dans le cas des premières molaires permamentes [18]. Ceci d'autant que les scellements doivent faire l'objet d'un suivi régulier pour intercepter leurs factures responsables de perte d'étanchéité et de développement de lésions carieuses à bas bruit.

\section{T2 Indication, protocole}

TEG1 Depuis 2010, la Haute Autorité de santé (HAS) recommande l'application semestrielle de vernis fluoré, en cario-prévention primaire, chez les patients à risque carieux élevé, une application trimestrielle n'ayant pas démontré une efficacté supérieure [19]. Si, en France, ces produits ne sont utilisés que par les chirurgiens-dentistes, dans d'autres pays (comme les États-Unis), ils peuvent aussi l'être par d'autres professionnels de santé (pédiatres, infirmières, hygiènistes dentaires...). Ils sont très simples à appliquer : il suffit de badigonner les dents de vernis avec un petit pinceau (ou une micro-brosse) et d'aider le produit à imprégner les espaces interdentaires grâce au passage de fil dentaire. La seule contrainte est qu'il ne faut ni boire ni manger dans les deux à quatre heures qui suivent (figure 3$)^{2}$.

TEG1 Les vernis fluorés, malgré leurs bons résultats, ne doivent pas être considérés isolément des autres mesures préventives telles qu'une bonne hygiène orale avec un dentifrice fluoré [20] et une correction des facteurs alimentaires.

\section{T2 Prévention secondaire}


TEG1 Les vernis fluorés peuvent aussi être utilisés en prévention secondaire, pour la reminéralisation des lésions carieuses non-cavitaires [21-23]. Des applications répétées au minimum trois fois sont nécessaires, mais la fréquence optimale d'application ne fait pas consensus [15]. En effet, la reminéralisation a été décrite en denture temporaire avec deux applications à T0 et quatre mois [24], mais aussi grâce à quatre applications successives à une semaine d'intervalle [25]. En denture permanente, elle a, par exemple, été rapportée pour deux applications à une semaine d'intervalle [23], quatre applications à une semaine d'intervalle [26], ainsi que pour des applications mensuelles pendant six mois [27].

\section{T1 Place des agents fluorés dans le cadre de la CCAM}

TEG1 À la suite de la décision de l'Union nationale des caisses d'assurance maladie (Uncam) relative à la liste des actes et prestations pris en charge par l'Assurance maladie du

18 décembre 2018, l'acte HBLD045 a été créé, au sous-paragraphe "07.02.02.04 - prophylaxie buccodentaire". Correspondant à une application de vernis fluoré sur les deux arcades dentaires, il est indiqué comme acte de prévention en cas de risque carieux élevé. Sa prise en charge (application unitaire : $25 €$ ) est limitée aux enfants de 6 à 9 ans présentant un risque carieux individuel (RCI) élevé, sur les deux arcades, deux fois par an maximum. Le texte officiel est paru le 16 février 2019 pour une application au $1^{\text {er }}$ avril 2019 [28].

TEG1 Cette décision représente une réelle évolution en prévention primaire, car si le code HBLD045 existait déjà dans la CCAM sous l'intitulé "application dentaire d'un vernis de reminéralisation sur une arcade", il ne faisait pas l'objet de tarification.

TEG1 Malgré la restriction d'âge par la codification et la tarification dans le cadre de la CCAM [28], les vernis fluorés doivent être indiqués, en prévention primaire, chez tous les patients à haut risque carieux quel que soit leur âge, avant 6 ans mais aussi au-delà de 9 ans [15-18]. Lorsqu'ils sont mis en œuvre à visée thérapeutique pour la reminéralisation de lésions amélaires non-cavitaires, deux séances annuelles sont inefficaces [15,21-27]. Hors cadre CCAM (en raison de l'âge et/ou de nombre d'applications nécessaires), la mise en œuvre des vernis fluorés peut être réalisée dans le cadre des actes hors-nomenclature soumis à l'édition d'un devis. Malgré une nette avancée en termes de prévention primaire, il existe malheureusement toujours un écart entre preuves scientifiques et CCAM [29].

\section{T1 Conclusion}

Dans le cadre de l'intervention minimale en cariologie, dentifrices et vernis fluorés sont les deux produits actuellement privilégiés en prévention primaire comme en prévention secondaire. Leur efficacité dépendra de la gestion du risque carieux qui sera contrôlée à l'occasion du suivi du patient. 


\section{Points à retenir}

- Le dosage en fluor des dentifrices dépend de l'âge du sujet et de son risque carieux.

- Un dentifrice fluoré doit être utilisé à tous les âges.

- L'application de vernis fluoré est effectuée par un chirurgien-dentiste.

- Elle est semestrielle chez un sujet à risque carieux élevé.

- L'utilisation d'un vernis fluoré est inutile chez un patient à risque carieux faible.

Sur 2 colonnes à droite après la puce de fin

\section{Notes}

${ }^{1}$ Grâce à la correction des mauvaises habitudes alimentaires et à l'utilisation biquotidienne de dentifrice de 1000 ppmF, les lésions non cavitaires occlusales de la deuxième molaire temporaire gauche ont été reminéralisées et la lésion carieuse cavitaire de la première molaire temporaire gauche a été contrôlée (arrêtée).

${ }^{2}$ Après le nettoyage des dents, le vernis d'au moins 22600 ppmF (Duraphat ${ }^{\circledR}$, Colgate) est appliqué. L'enfant devra attendre au moins deux heures pour manger et boire. II ne se brossera pas les dents le jour de l'application pour laisser la couche au maximum au contact des dents.

\section{Références}

[1] Kassebaum N, Smith A, Bernabé E. Global, regional, and national prevalence, incidence, and disability-adjusted life years for oral conditions for 195 countries, 1990-2015: A systematic analysis for the global burden of diseases, injuries, and risk factors. J Dent Res. 2017;96(4):380-7.

[2] Kassebaum NJ, Bernabé E, Dahiya M et al. Global burden of untreated caries: a systematic review and metaregression. J Dent Res. 2015;94(5):650-8.

[3] Muller-Bolla M, Doméjean S. Sucres et santé bucco-dentaire. Cah Nutr Diet. 2018;53:341-6.

[4] Marinho VCC. Applying prescription-strength home-use and professionally applied topical fluoride products may benefit people at high risk for caries - the American Dental Association (ADA) 2013 clinical practice guideline recommendations. J Evid-Based Dent Pract. 2014;14(3):120-3.

[7] Decup F, Muller-Bolla M, Chouvin $\mathrm{M}$ et al. Dentifrices et vernis fluorés : bilan des connaissances pour une bonne pratique clinique. Clinic. 2018;39:329-36.

[8] Marinho VC, Higgins JP, Sheiham A et al. Fluoride toothpastes for preventing dental caries in children and adolescents. Cochrane Database Syst Rev. 2003;1:CD002278. 
[9] Walsh T, Worthington HV, Glenny AM et al. Fluoride toothpastes of different concentrations for preventing dental caries in children and adolescents. Cochrane Database Syst Rev. 2010;1:CD007868.

[10] Doméjean S, Muller-Bolla M, Featherstone J. Prevention in dental practice. In: Understanding dental caries from pathogenesis to prevention and therapy (Goldberg M). Cham (Suisse): Springer International Publishing AG; 2016.

[11] Agence française de sécurité sanitaire et des produits de Santé (Affsaps). Utilisation du fluor dans la prévention de la carie dentaire avant l'âge de 18 ans. Octobre 2008. https://ansm.sante.fr/var/ansm_site/storage/original/application/7db1d82db7f5636b56170f59e844 dd3a.pdf

[5] Wong M, Glenny A, Tsang B et al. Topical fluoride as a cause of dental fluorosis in children. Cochrane Database Syst Rev. 2010;1:(CD007693).

[12] Collège des enseignants d'odontologie pédiatrique (CEOP). Guide d'odontologie pédiatrique. La clinique par la preuve. Malakoff: Cdp; 2018.

[6] Robert D, Courson F, Muller-Bolla M. Les enfants doivent-ils utiliser des brosses à dents manuelles ou électriques ? Rev Francoph Odontol Pediatr. 2016;11(4):150-4.

[13] Mijan M, de Amorim RG, Leal SC et al. The 3.5-year survival rates of primary molars treated according to three treatment protocols: a controlled clinical trial. Clin Oral Investig. 2014;18(4):10619.

[14] Innes NPT, Frencken JE, Bjørndal L et al. Managing carious lesions: Consensus recommendations on terminology. Adv Dent Res. 2016;28(2):49-57.

[15] Marinho VCC, Worthington HV, Walsh T et al. Fluoride varnishes for preventing dental caries in children and adolescents. Cochrane Database Syst Rev. 2013;7:CD002279.

[16] Ahovuo-Saloranta A, Forss H, Walsh T et al. Pit and fissure sealants for preventing dental decay in the permanent teeth. Cochrane Database Syst Rev. 2017;7:CD001830.

[17] Ahovuo-Saloranta A, Forss $\mathrm{H}$, Hiiri A et al. Pit and fissure sealants versus fluoride varnishes for preventing dental decay in the permanent teeth of children and adolescents. Cochrane Database Syst Rev. 2016;1:CD003067.

[18] Chestnutt IG, Playle R, Hutchings S et al. Fissure Seal or Fluoride Varnish? A Randomized Trial of Relative Effectiveness. J Dent Res. 2017;96(7):754-61.

[19] Haute Autorité de santé. Stratégies de prévention de la carie dentaire. Mars 2010. www.hassante.fr/portail/upload/docs/application/pdf/2010-

10/corriges_synthese_carie_dentaire_version_postcollege-10sept2010.pdf

[20] Marinho VCC, Higgins JPT, Sheiham A et al. Combinations of topical fluoride (toothpastes, mouthrinses, gels, varnishes) versus single topical fluoride for preventing dental caries in children and adolescents. Cochrane Database Syst Rev. 2004;1:CD002781. 
[21] Lenzi TL, Montagner AF, Soares FZM et al. Are topical fluorides effective for treating incipient carious lesions? A systematic review and meta-analysis. J Am Dent Assoc. 2016;147: 84-91 e81.

[22] Gao SS, Zhang S, Mei ML et al. Caries remineralisation and arresting effect in children by professionally applied fluoride treatment - a systematic review. BMC Oral Health. 2016;16:12.

[23] Restrepo M, Bussaneli D, Jeremias F et al. Control of white spot lesions with use of fluoride varnish or chlorhexidine gel during orthodontic treatment - a randomized clinical trial. J Clin Pediatr Dent. 2016;40(4):274-80.

[24] Autio-Gold J, Courts F. Assessing the effect of fluoride varnish on early enamel carious lesions in the primary dentition. J Am Dent Assoc. 2001;132(9):1247-53.

[25] de Amorim R, Leal SC, Bezerra AC et al. Association of chlorhexidine and fluoride for plaque control and white spot lesion remineralization in primary dentition. Int J Paediatr Dent. 2008;18(6):446-51.

[26] Ferreira J, Aragao A, Rosa A et al. Therapeutic effect of two fluoride varnishes on white spot lesions: a randomized clinical trial. Braz Oral Res. 2009;23(4):446-51.

[27] Du M, Cheng N, Tai B et al. Randomized controlled trial on fluoride varnish application for treatment of white spot lesion after fixed orthodontic treatment. Clin Oral Investig. 2012;16(2):4638

[28] Décision du 18 décembre 2018 de l'Union nationale des caisses d'Assurance maladie relative à la liste des actes et prestations pris en charge par l'assurance maladie. Journal officiel de la République française du 16 février 2019.

[29] Doméjean S, Deschamps $M$, Espinasse $L$ et al. Intervention minimale en cariologie : I'intégration indispensable à la CCAM. Info Dent. 2018;31:33-9.

Déclaration de liens d'intérêts

Les auteurs déclarent ne pas avoir de liens d'intérêts.

\section{Illustration}

\section{Mul_illus1}

(C) M. Muller-Bolla

A intégrer dans l’encadré 1 sans légende

\section{Figures}




\section{Mul_fig1}

Figure 1. Quantité de dentifrice fluoré à déposer sur la brosse à dents d'un enfant porteur d'une denture temporaire (à l'état de trace jusqu'à 2 ans, puis d'une quantité équivalente à un petit pois de 2 à 6 ans).

Sur 2 colonnes ou 1 colonne + marge près de son appel

\section{Mul_fig2}

Figure 2. Lésions carieuses contrôlées (arrêtées) grâce à l'utilisation biquotidienne de dentifrice à $1000 \mathrm{ppmF}$.

(C) M. Muller-Bolla

Sur 2 colonnes ou 1 colonne + marge près de son appel

\section{Mul_fig3}

Figure 3. Application professionnelle de vernis fluoré pour reminéraliser les lésions non cavitaires intéressant la face occlusale de la deuxième molaire temporaire droite et la face proximale de la première molaire temporaire.

(C) M. Muller-Bolla

Sur 2 colonnes ou 1 colonne + marge près de son appel 


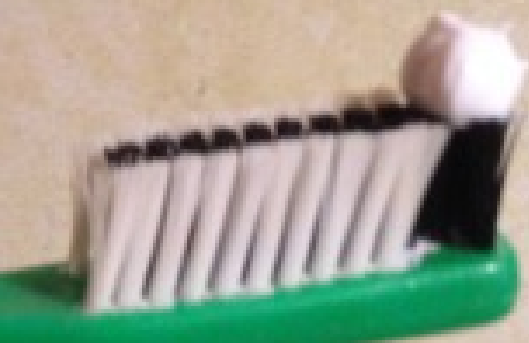




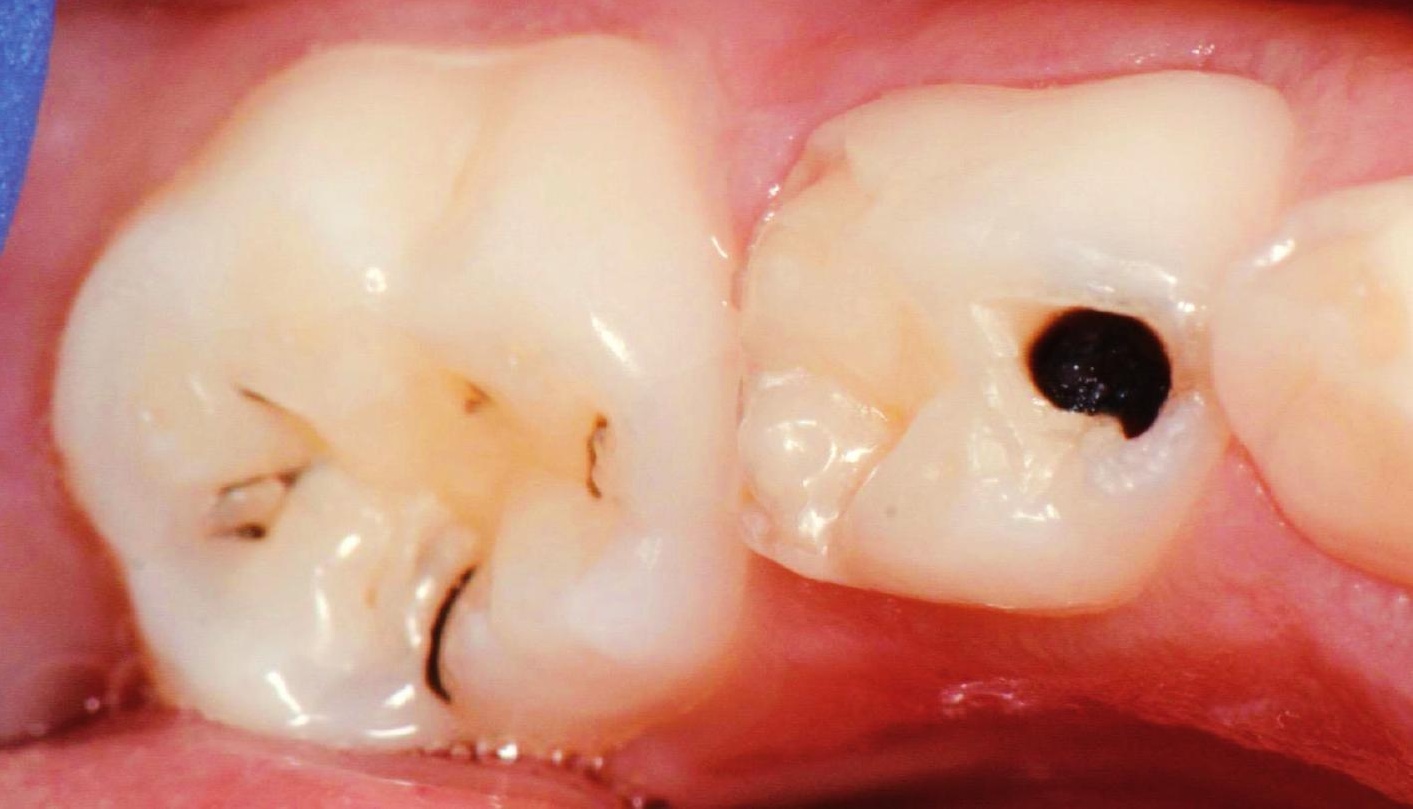




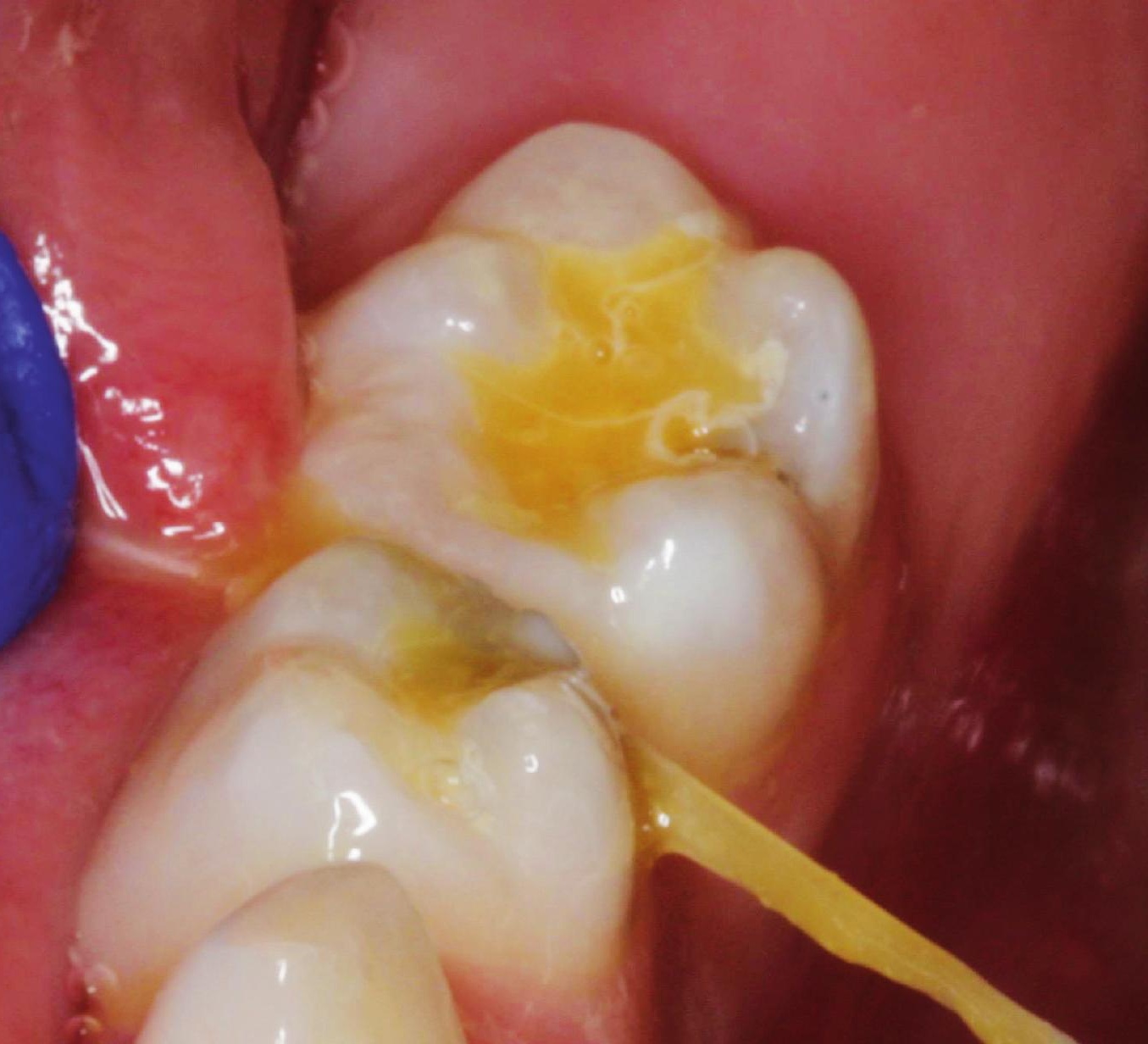

